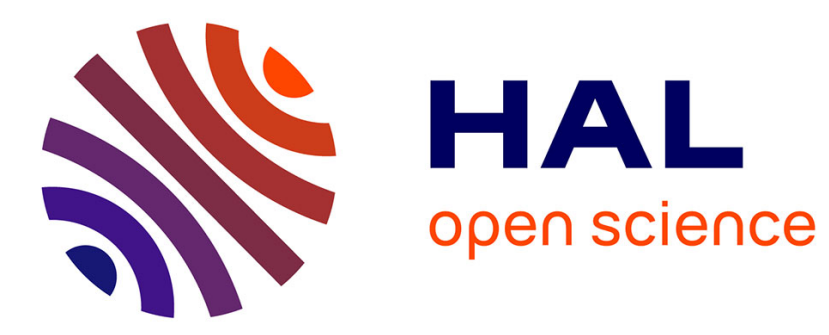

\title{
Ultrafast dynamics of the coherences in a quantum Hall system
}

Keshav M. Dani, Jérôme Tignon, Michael Breit, Daniel S. Chemla, Eleftheria Kavousanaki, Ilias E. Perakis

\section{- To cite this version:}

Keshav M. Dani, Jérôme Tignon, Michael Breit, Daniel S. Chemla, Eleftheria Kavousanaki, et al.. Ultrafast dynamics of the coherences in a quantum Hall system. Physical Review Letters, 2006, 97, pp.057401. hal-00110001

\section{HAL Id: hal-00110001 https://hal.science/hal-00110001}

Submitted on 26 Oct 2006

HAL is a multi-disciplinary open access archive for the deposit and dissemination of scientific research documents, whether they are published or not. The documents may come from teaching and research institutions in France or abroad, or from public or private research centers.
L'archive ouverte pluridisciplinaire HAL, est destinée au dépôt et à la diffusion de documents scientifiques de niveau recherche, publiés ou non, émanant des établissements d'enseignement et de recherche français ou étrangers, des laboratoires publics ou privés. 


\title{
Ultrafast dynamics of coherences in the quantum Hall system
}

\author{
K.M. Dani ${ }^{1,2}$, J. Tignon ${ }^{3}$, M. Breit ${ }^{2}$ and D.S. Chemla ${ }^{1,2}$ \\ ${ }^{1}$ Department of Physics, University of California at Berkeley, \\ ${ }^{2}$ Materials Sciences Division, E.O. Lawrence Berkeley National Laboratory, Berkeley, California 94720, and \\ ${ }^{3}$ Laboratoire Pierre Aigrain, Ecole Normale Supérieure, F-75005 Paris, France. \\ E.G. Kavousanaki and I.E. Perakis \\ Institute of Electronic Structure \& Laser, Foundation for Research \& Technology-Hellas, \\ and Department of Physics, University of Crete, Heraklion, Greece.
}

(Dated: July 24, 2006)

\begin{abstract}
Using three-pulse four-wave-mixing optical spectroscopy, we study the ultrafast dynamics of the quantum Hall system. We observe striking differences as compared to an undoped system, where the 2D electron gas is absent. In particular, we observe a large off-resonant signal with strong oscillations. Using a microscopic theory, we show that these are due to many-particle coherences created by interactions between photoexcited carriers and collective excitations of the 2D electron gas. We extract quantitative information about the dephasing and interference of these coherences.
\end{abstract}

PACS numbers: 78.47.+p, 42.50.Md, 73.20.Mf, 78.67.De

The quantum Hall effects [1, 2, 3] arise in a cold two-dimensional electron gas (2DEG) in a perpendicular magnetic field. They result from Coulomb correlations among the ground state 2DEG electrons that populate the highly degenerate Landau level states. Previous transport and optics experiments have studied the properties of this incompressible electron fluid and its elementary excitations [2, 4]. Of interest here are the collective charge excitations called magnetoplasmons (MP). Unlike for zero magnetic field [5], in the quantum Hall regime the correlations and incompressible ground state result in a pronounced magnetoroton minimum [3]. Despite their large momenta, magnetorotons dominate the inelastic light scattering [2, 3, 4]. However, such experiments cannot access MP dynamics. Moreover, they cannot access the early timescales required to observe $\mathrm{co}$ herent effects in the quantum Hall system. Coherences play a central role in several quantum mechanical systems 6, 7, 8, 9, 10]. Recent proposals for quantum computing point out the need to study the coherent dynamics of the quantum Hall system [11]. Ultrafast non-linear optics is just beginning to explore these phenomena $12,13,14]$.

Ultrafast four-wave-mixing (FWM) spectroscopy is well suited for studying coherent dynamics 15. It has demonstrated that Coulomb interactions in undoped semiconductor quantum wells are crucial to this dynamics and lead to exciton-exciton and carrier-phonon interactions, non-Markovian memory effects, etc. 15, 16, 17, 18]. However, in undoped quantum wells the lowest electronic excitations are high energy interband transitions that react almost instantaneously to the photoexcited carriers [19]. The ground state can then be considered as rigid, providing only the band structure and dielectric screening. Consequently, Coulomb correlations only occur among photoexcited carriers. In contrast, in doped quantum wells, the presence of a 2DEG leads to strong
Coulomb correlations in the ground state itself, resulting in long-range charge and spin order at sufficiently low temperatures. This order determines the 2DEG reaction to photoexcitation. Therefore, one must distinguish the effects of this cold 2DEG from those due to the nonequilibrium photoexcited electron gas. The differences manifest themselves in the ultrafast nonlinear response.

In this letter we use three-pulse FWM spectroscopy to probe simultaneously the intra- and inter-band coherent dynamics of the quantum Hall system. For low temperatures, weak photoexcitation, and high magnetic fields, we observe striking qualitative differences in the coherent response of doped and undoped quantum wells. We see a large off-resonant signal from the lowest Landau level (LL0), with strong oscillations versus pulse time delay in Fig.1a. Using a microscopic theory 13, 14, we show that this LL0 signal is dominated by the interference between many-particle coherences created by the interaction of photoexcited carriers with the 2DEG. Photoexcited excitons $(\mathrm{X})$ scatter to $\mathrm{X}+\mathrm{MP}$ states (i.e. states with a photoexcited exciton and a MP excitation of the 2DEG). Within ultra-short time scales, this scattering creates a coherence between the above states - a X $\leftrightarrow \mathrm{X}+\mathrm{MP}$ manyparticle coherence. We show that the oscillations are due to the interference between FWM contributions from these coherences and put an upper bound on their dephasing rate. The presence of the $\mathrm{X} \leftrightarrow \mathrm{X}+\mathrm{MP}$ coherences indicates a breakdown of the semiclassical (Boltzmann) picture [15, 16, 17] of irreversible scattering.

In our experiment, we excite the quantum Hall system with three $100 \mathrm{fs} \sigma_{+}$-polarized pulses along directions $\mathbf{k}_{1}$, $\mathbf{k}_{2}$, and $\mathbf{k}_{3}$. Pulses $\mathbf{k}_{1}$ and $\mathbf{k}_{2}\left(\mathbf{k}_{3}\right)$ are separated by a time delay $\Delta t_{12}\left(\Delta t_{13}\right)$, where pulse $\mathbf{k}_{1}$ arrives first for negative values of the delay. The FWM response is obtained in the background-free direction $\mathbf{k}_{1}+\mathbf{k}_{2}-\mathbf{k}_{3}$. Using an interference filter of bandwidth $2 \mathrm{meV}$, we spectrally re- 

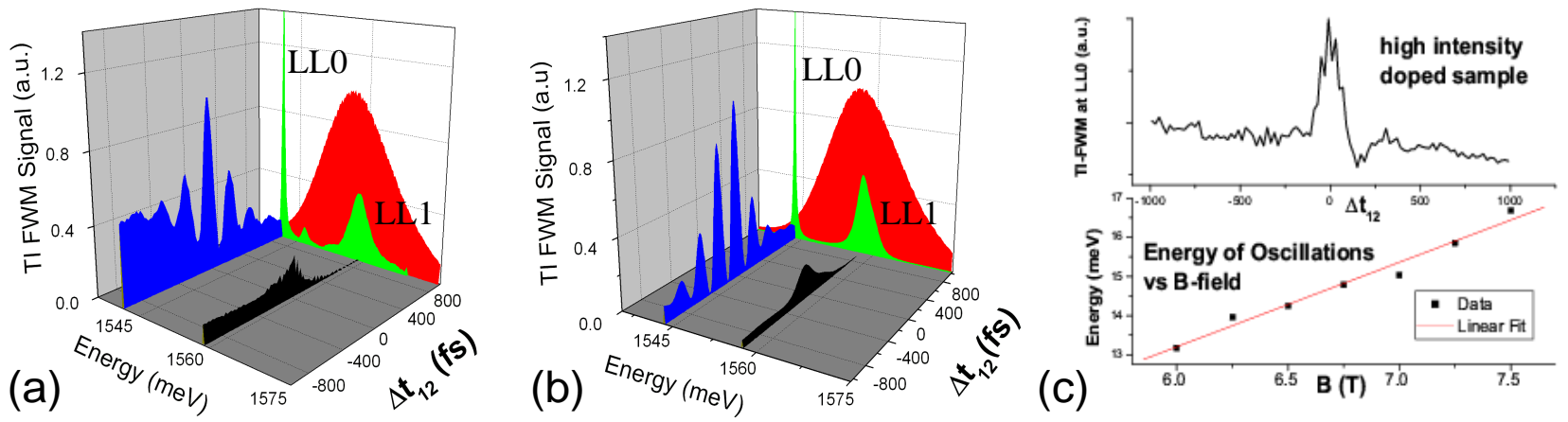

FIG. 1: Three-pulse FWM signal for the doped quantum well along the $\Delta t_{12}$ axis $\left(\Delta t_{13}=0\right)$ for low excitation intensity (a) experiment and (b) theory. (Backpanel: Linear absorption and optical pulse.) (c) Top panel: high excitation intensity of doped quantum well along the $\Delta t_{12}$ axis. Bottom panel: B-field dependence of the oscillation frequency.

solve the response so as to separate out the contribution from each Landau level. We then measure the intensity from each Landau level as a function of the time delays. In particular, we measure along the $\Delta t_{12}$ axis $\left(\Delta t_{13}=0\right)$ or the $\Delta t_{13}$ axis $\left(\Delta t_{12}=0\right)$. For the $\Delta t_{13}$ axis, pulses $\mathbf{k}_{1}$ and $\mathbf{k}_{2}$ arrive together. In this case, the physics is similar to 2-pulse FWM 12], where $\mathbf{k}_{1}$ and $\mathbf{k}_{2}$ are degenerate. The $\Delta t_{13}$ axis thus reflects the dynamics of the optical polarizations (inter-band) [15]. On the other hand, the $\Delta t_{12}$ axis gives access to new dynamics, like the temporal evolution of the $\mathrm{X} \leftrightarrow \mathrm{X}+\mathrm{MP}$ coherence (intra-band).

We investigated a modulation-doped quantum well structure consisting of 10 periods of $12 \mathrm{~nm}$ GaAs wells and $42 \mathrm{~nm} \mathrm{AlGaAs}$ barrier layers with Si doped at their centers. The doped carrier (2DEG) density was $2.1 \times 10^{11}$ $\mathrm{cm}^{-2}$ and the low temperature mobility of the sample was $\sim 10^{5} \mathrm{~cm}^{2} / \mathrm{Vs}$. The sample was kept at $1.5-4^{\circ} \mathrm{K}$ in a split-coil magneto-optical-cryostat. A perpendicular magnetic field $(\mathrm{B}=0-7 \mathrm{~T})$ was applied along the growth direction of the quantum well. The bulk of our measurements were performed at $\mathrm{B}=7 \mathrm{~T}$ (filling factor $\nu=1.3$ ). To isolate the effects of the interactions, we measured the FWM signal from the first Landau level (LL0) while largely exciting the second Landau level (LL1) (LL1:LL0 population ratio at least 10:1, see back panel of Fig. 1a). We then compared the LL0 signal from the doped quantum well to a similar undoped one (without a 2DEG). We also compared low and high intensity measurements. For low intensity, the photoexcited carrier density $\left(5 \times 10^{9}\right.$ $\mathrm{cm}^{-2}$ ) was kept much smaller than the 2DEG density in order to weakly perturb the quantum Hall system. For high intensity, the two densities were comparable, so the photoexcited carrier contribution was strong.

For large LL1/LL0 photoexcitation ratio, the phase space filling contribution to LL0 is very small. The LL0 signal then comes from LL1-LL0 coupling due to (i) exciton-exciton interactions 20], (ii) inter-Landau level coherences of the photoexcited carriers, and (iii) X-2DEG interactions. Only the first two contribute in undoped semiconductors. Since the LL0 signal in the undoped quantum well is small (Fig. 2b), these contributions are weak. In contrast, the doped quantum well shows a large LL0 signal (Figs. 1a, 2a). This signal diminishes for small magnetic fields, $\nu>2$. It is also small for high intensities, when the photoexcited carriers dominate over the 2DEG (Fig. 2c). We therefore conclude that our LL0 signal is due to LL0-LL1 coupling via X-2DEG interactions.

Along the $\Delta t_{13}$ axis, we observe striking differences between the doped and undoped quantum well similar to two-pulse FWM 12]. However, three-pulse FWM along the $\Delta t_{12}$ axis provides new information about the dynamics of $\mathrm{X} \leftrightarrow \mathrm{X}+\mathrm{MP}$ coherences that is not accessible with two-pulse FWM. Fig. 1a shows the FWM signal in the doped quantum well along the $\Delta t_{12}$ axis for low photoexcitation, high magnetic field, and low temperature. Besides the large transfer of signal strength to LL0, we observe strong $\Delta t_{12}$ oscillations only at LL0. There are no oscillations at LL1 or in the $\Delta t_{13}$ axis. The oscillation frequency is comparable to the inter-Landau level energy spacing and increases linearly with the B-field (bottom panel of Fig. 1c). The oscillation decay rate for both positive and negative $\Delta t_{12}$ is comparable to the sum of the LL0 and LL1 dephasing rates extracted from Fig. 2a. With increasing photoexcitation intensity, these $\Delta t_{12}$ oscillations disappear quickly, even before the decay of the overall signal changes significantly (top panel of Fig. 1c).

Theory: To understand the non-linear response of the quantum Hall system, we use a model derived from the many-body theory of Ref. [14]. This theory goes beyond the Dynamics Controlled Truncation Scheme (DCTS) 21] used to study correlations in undoped semiconductors. The DCTS relies on the correspondence between electron-hole pairs created or destroyed and the sequence of photons absorbed or emitted. However, if carriers are present in the system before excitation (e.g. doped quantum well), this correspondence breaks down and the DCTS fails. We extend the DCTS by tracking only the photoexcited holes created (destroyed) with the photons 

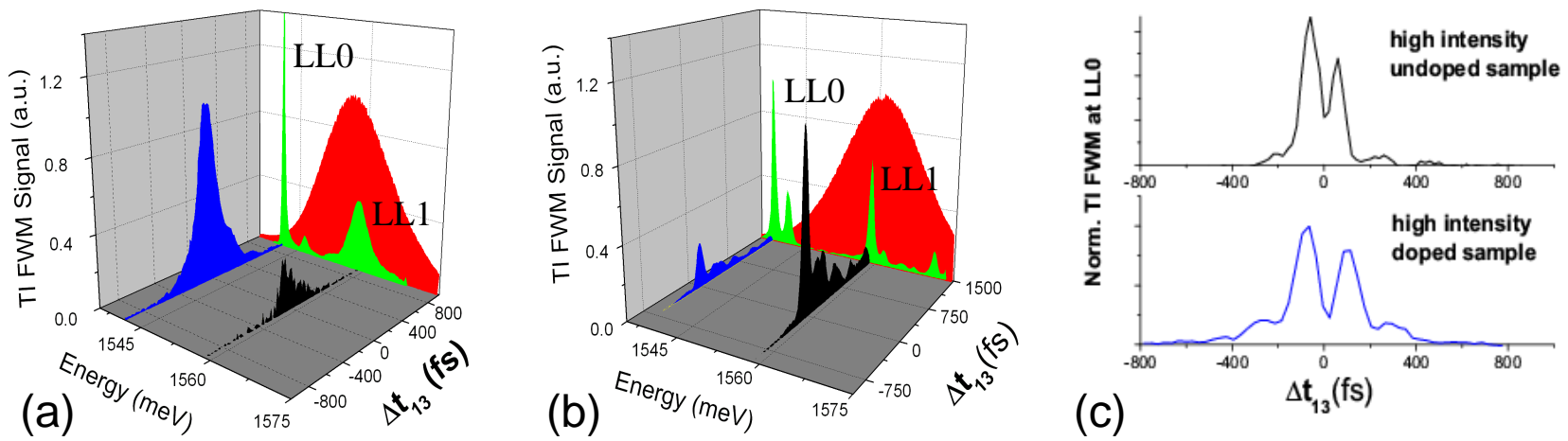

FIG. 2: Three-pulse FWM signal for low excitation intensity along the $\Delta t_{13}$ axis $\left(\Delta t_{12}=0\right)(\mathrm{a})$ doped and (b) undoped quantum well. (Backpanel: Linear absorption and optical pulse.) (c) Comparison of doped and undoped quantum wells for high excitation intensity along the $\Delta t_{13}$ axis.

absorbed (emitted). Thus we account for the presence of the 2DEG prior to photoexcitation and can treat the $\mathrm{X}-2 \mathrm{DEG}$ interactions. There is a further challenge in studying the optical response of a 2DEG. In an undoped system, the excited states can be written as products of distinct phonon and $\mathrm{X}$ states. However, in the doped system, both the 2DEG excitations and the Xs are made up of electrons. Thus exchange effects complicate a simple factorization. We overcome this difficulty by introducing a basis of correlated X-2DEG states 14].

The third order optical polarization is determined by phase space filling and an interaction induced contribution. By minimizing the optical pulse overlap at LLO, phase space filling contributions at LL0 are suppressed. The interaction induced contributions are described by a density matrix $\langle\hat{Y}\rangle$ 13, 14]. This includes excitonexciton interactions, which occur in the undoped quantum wells as well, and X-2DEG interactions. In Refs. [13, 14] we decomposed $\langle\hat{Y}\rangle$ into three parts: (i) coherent exciton-exciton interactions as in the undoped quantum well; (ii) contributions from intraband coherences and densities, including the $\mathrm{X} \leftrightarrow \mathrm{X}+\mathrm{MP}$ coherences; (iii) a correlated contribution governed by the time evolution of the X+MP states. The latter dephases rapidly and mainly contributes to the LL1 exciton linewidth 12, 13].

For simplicity we consider a spin- $\uparrow$ polarized ground state 2DEG (realized for $\nu=1$ ). We then excite spin$\downarrow$ electrons with $\sigma_{+}$light. Due to quantitative uncertainties arising from disorder, valence band mixing, and higher Landau levels, we determined the independent parameters by fitting to the experimental linear absorption (back panel of Fig. 1a). Our conclusions are not sensitive to their precise values.

The $\mathrm{X} \leftrightarrow \mathrm{X}+\mathrm{MP}$ coherence that dominates our results, denoted by $M_{0}$ from now on, is created by the scattering of the LL1 exciton to LL0 with the emission of a MP. Note that, due to the degeneracy of the LL1 and LL0+MP states, the coherence $M_{0}$ has small energy compared to the inter-Landau level spacing. It can be created by pulses $1-3\left(M_{0}^{13}\right)$ or pulses $2-3\left(M_{0}^{23}\right)$. The corresponding resonant FWM contribution to LL0 in our calculation comes from $P_{0} M_{0}^{*}$, where $P_{0}$ is the LL0 polarization created by the third pulse to probe the $M_{0}$ coherence [14]. Details of the calculation will be presented elsewhere.

Origin of oscillations: Fig. 3a shows a schematic describing the contribution from the $M_{0}^{13}$ coherence. Pulses $\mathbf{k}_{1}$ and $\mathbf{k}_{3}$ arrive simultaneously in the sample to create a density of excitons in LL1. These excitons scatter into LL0 with the excitation of a MP, thereby creating the coherence $M_{0}^{13}$. This coherence evolves for a time $\left|\Delta t_{12}\right|$, accumulating negligible phase due to the small $M_{0}$ energy. It is then probed by a $P_{0}$ polarization created by $\mathbf{k}_{2}$, resulting in a FWM signal in $\mathbf{k}_{1}+\mathbf{k}_{2}-\mathbf{k}_{3}$. Due to the symmetry of $\mathbf{k}_{1}$ and $\mathbf{k}_{2}$ in the $\mathbf{k}_{1}+\mathbf{k}_{2}-\mathbf{k}_{3}$ signal, we also have a process where $\mathbf{k}_{2}$ and $\mathbf{k}_{3}$ create the coherence $M_{0}$ (i.e. $M_{0}^{23}$ ). This is then probed by $\mathbf{k}_{1}$. However, one must keep track of the time delays. Thus, as shown in Fig. $3 \mathrm{~b}$, now $\mathbf{k}_{1}$ and $\mathbf{k}_{3}$ arrive together with $\mathbf{k}_{3}$ contributing a LL1 polarization and $\mathbf{k}_{1}$ contributing a LL0 polarization. These polarizations evolve in the sample for a time $\left|\Delta t_{12}\right|$ and accumulate a phase of $\left(\omega_{0}-\omega_{1}\right) \Delta t_{12}$ (where $\hbar \omega_{n}$ is the energy of the $n^{\text {th }}$ Landau level). Pulse $\mathbf{k}_{2}$ then creates the $M_{0}^{23}$ coherence with the decaying LL1 polarization from $\mathbf{k}_{3} . M_{0}^{23}$ is instantaneously probed by the decaying LL0 polarization created earlier by $\mathbf{k}_{1}$, resulting in a FWM signal with the accumulated phase $\left(\omega_{0}-\omega_{1}\right) \Delta t_{12}$. The first process (due to $M_{0}^{13}$ ) contributes to the FWM signal for $\left|\Delta t_{12}\right|$ shorter than the decay time of $M_{0}^{13}$, while the second process (due to $M_{0}^{23}$ ) contributes for $\left|\Delta t_{12}\right|$ shorter than the polarization decay times. Within the shortest time interval of the two, the contributions from $M_{0}^{13}$ and $M_{0}^{23}$ will interfere with each other, resulting in oscillations at the inter-Landau level frequency $\omega_{0}-\omega_{1}$ along the $\Delta t_{12}$ axis. These do not contribute along the $\Delta t_{13}$ axis, or in two-pulse FWM [12]. Fig. 4 shows the numerical calculation of the $M_{0}^{13}, M_{0}^{23}$ contributions and the interference between the two when considered together $\left(M_{0}\right)$. The signal from $M_{0}$ dominates 
(a)

(b)

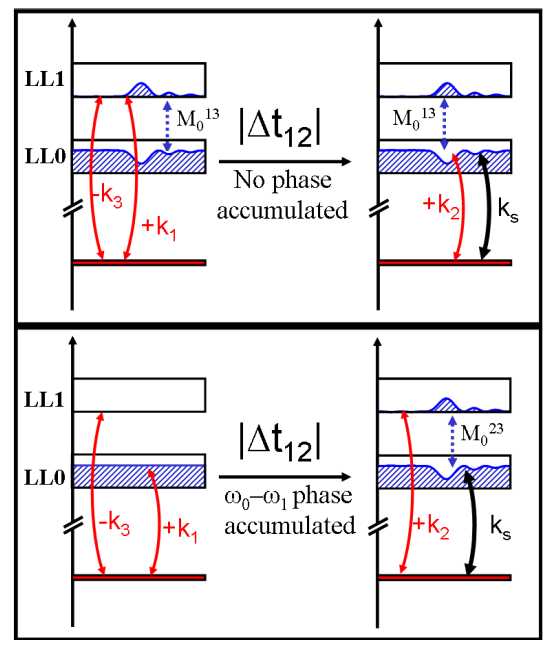

FIG. 3: Third-order process contributing to the FWM signal (in the direction $\mathbf{k}_{s}=\mathbf{k}_{1}+\mathbf{k}_{2}-\mathbf{k}_{3}$ ) due to (a) $M_{0}^{13}$ (b) $M_{0}^{23}$.

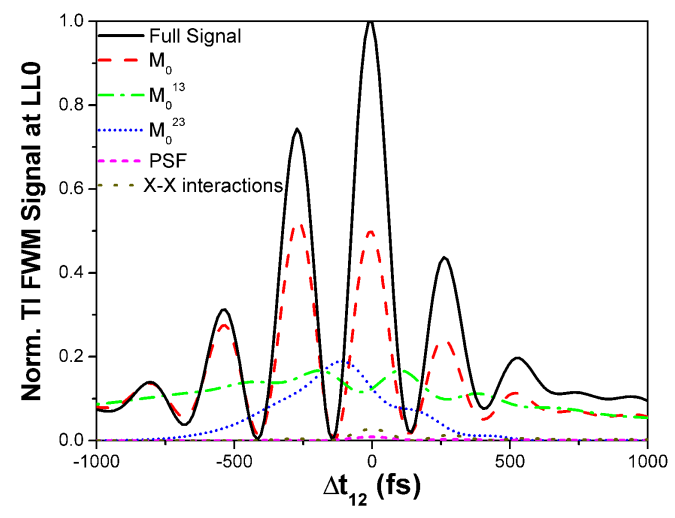

FIG. 4: Numerical calculation of the LL0 FWM signal for the $\Delta t_{12}$ axis due to different processes.

the full FWM signal. Contributions from phase space filling and $\mathrm{X}-\mathrm{X}$ interactions are negligible. Fig. 1b shows the full numerical calculation which reproduces well the experimental features in the coherent regime.

To interpret the decay of the above oscillations, we assign effective dephasing rates $\Gamma_{0}$ and $\Gamma_{1}$ to the LL0 and LL1 polarizations and assume a $M_{0}$ dephasing rate of $\gamma_{0}$. The decay of oscillations for $\Delta t_{12}>0$ is $\Gamma_{0}+\Gamma_{1}$, whereas for $\Delta t_{12}<0$ we get a decay of $\Gamma_{0}+\Gamma_{1}+\gamma_{0}$. As the experiment shows, the decay of oscillations is nearly equal for positive and negative $\Delta t_{12}$. Thus, $\gamma_{0} \ll \Gamma_{0}+\Gamma_{1}$, i.e. the $\mathrm{X} \leftrightarrow \mathrm{X}+\mathrm{MP}$ coherence dephases over a time interval of $1 / \gamma_{0} \gg 1 /\left(\Gamma_{0}+\Gamma_{1}\right) \sim 300$ fs.

In conclusion, by using three-pulse FWM spectroscopy, we access for the first tme the full coherent dynamics of the quantum Hall system. We see a large off-resonant signal with strong oscillations only along the $\Delta t_{12}$ axis. Using a microscopic many-body theory, we show that the signal is due to many-particle coherences created via the non-instantaneous interactions of photoexcited carriers and MPs. The oscillations are due to the interference of different FWM contributions of these coherences. Finally, we put an upper bound to the decay rate of the $\mathrm{X} \leftrightarrow \mathrm{X}+\mathrm{MP}$ coherence. The combination of ultrafast nonlinear spectroscopy and quantum Hall physics initiates a new field of coherent quantum Hall dynamics.

We thank N.A. Fromer, S. Cundiff and J. Wang for discussions. This work was supported by the Office of Basic Energy Sciences of the US Department of Energy, the France-Berkeley Foundation, and by the EU Research Training Network HYTEC.

[1] H. L. Stormer, D. C. Tsui, and A. C. Gossard, Rev. Mod. Phys. 71, S298 (1999).

[2] Perspectives in Quantum Hall Effects, S. Das Sarma and A. Pinczuk (eds.), (Wiley, New York, 1977).

[3] D. Yoshioka D, The Quantum Hall Effect (SpringerVerlag, New York, 2002).

[4] A. Pinczuk et. al., Phys. Rev. Lett. 61, 2701 (1988); ibid 70, 3983 (1993); C.F. Hirjibehedin et. al. Phys. Rev. Lett. 95, 066803 (2005); J. P. Eisenstein, Solid State Commun. 117, 123 (2001).

[5] C. W. Luo et. al., Phys. Rev. Lett. 92, 047402 (2004); T. V. Shahbazyan et. al., Phys. Rev. Lett. 84, 2006 (2000); and references therein.

[6] V. M. Axt and T. Kuhn, Rep. Prog. Phys. 67, 433 (2004).

[7] H. A. Fertig and G. Murthy, Phys. Rev. Lett. 95156802 (2005).

[8] K. J. Boller, A. Imamoglu and S. E. Harris, Phys. Rev. Lett. 66, 2593 (1991).

[9] A. S. Zibrov et al, Phys. Rev. Lett. 75001499 (1995).

[10] M. E. Donovan et al., Phys. Rev. Lett. 87, 237402 (2001); K. B. Ferrio and D. G. Steel, Phys. Rev. Lett. 80, 786 (1998); G. Bartels et. al., Phys. Rev. B 55, 16404 (1997).

[11] S. Das Sarma, M. Freedman, and C. Nayak, Phys. Rev. Lett. 94, 166802 (2005).

[12] N. A. Fromer et al., Phys. Rev. Lett. 89, 067401 (2002); ibid 83, 4646 (1999); Phys. Rev. B 66, 205314 (2002).

[13] A. T. Karathanos et. al., Phys. Rev. B 67, 035316 (2003).

[14] I. E. Perakis and E. G. Kavousanaki, Chem Phys. 318, 118 (2005).

[15] D. S. Chemla and J. Shah, Nature 411, 549 (2001).

[16] W. Schäfer and M. Wegener, Semiconductor Optics and Transport Phenomena (Springer, Berlin, 2002).

[17] H. Haug and A. P. Jauho, Quantum Kinetics in Transport and Optics of Semiconductors (Springer, Heidelberg, 1996); Q. T. Vu et. al., Phys. Rev. Lett. 85, 3508 (2000); R. Huber et. al., Phys. Rev. Lett. 94, 027401 (2005).

[18] F. Rossi and T. Kuhn, Rev. Mod. Phys. 74, 895 (2002).

[19] S. Louie in Computational Materials Sciences, edited by C. Y. Fong (World Scientific, Singapore, 1998); L. J. Sham, Phys. Rev. 150, 720 (1966).

[20] C. Stafford, S. Schmitt-Rink, and W. Schäfer, Phys. Rev. B 41, 10000 (1990); M. W. Wu and H. Haug, Phys. Rev. B 58, 13060 (1998).

[21] V. M. Axt, K. Victor, and A. Stahl, Phys. Rev. B 53, 7244 (1996); V. M. Axt and S. Mukamel, Rev. Mod. Phys. 70, 145 (1998). 